\title{
P0014
}

\section{COLOUR DIFFERENCE DISCRIMINATIONS OF YOUNG AND OLD OBSERVERS BASED ON DIFFERENT DISPLAYS}

\author{
Yonghui Xi et al.
}

DOI 10.25039/x46.2019.PO014

from

CIE x046:2019

\section{Proceedings}

of the

29th CIE SESSION

Washington D.C., USA, June 14 - 22, 2019

(DOI 10.25039/x46.2019)

The paper has been presented at the 29th CIE Session, Washington D.C., USA, June 14-22, 2019. It has not been peer-reviewed by CIE.

(C) CIE 2019

All rights reserved. Unless otherwise specified, no part of this publication may be reproduced or utilized in any form or by any means, electronic or mechanical, including photocopying and microfilm, without permission in writing from CIE Central Bureau at the address below. Any mention of organizations or products does not imply endorsement by the CIE.

This paper is made available open access for individual use. However, in all other cases all rights are reserved unless explicit permission is sought from and given by the CIE.

CIE Central Bureau

Babenbergerstrasse 9

A-1010 Vienna

Austria

Tel.: +4317143187

e-mail: ciecb@cie.co.at

www.cie.co.at 


\title{
COLOUR DIFFERENCE DISCRIMINATIONS OF YOUNG AND OLD OBSERVERS BASED ON DIFFERENT DISPLAYS
}

\author{
Xi, Y.H., He, R.L., Guo, C.L., Huang, M. \\ Beijing Institute of Graphic Communication, Beijing, CHINA \\ huangmin@bigc.edu.cn
}

DOI 10.25039/x46.2019.PO014

\begin{abstract}
In order to investigate the influences of the displays with different spectral primaries on observers' colour discrimination, two displays (IPAD and Qauto-220ex) with different RGB spectral primaries were selected and CIE recommended 5 colours (gray, red, yellow, green, blue) were presented on the IPAD display as the target samples. 30 young (aged from 20 to 27) and 17 old observers (aged from 61 to 74 ) were organized to carry out the colour matching experiments. The obtained results indicated that colorimetric values matched by observers of different ages are quite different, which have different distributions when plotted on $a^{*}{ }_{10} b^{*} 10$ plane and $\mathrm{L}^{*}{ }_{10} \mathrm{C}^{*}{ }_{10}$ plane.
\end{abstract}

Key Words: Colour Difference Discrimination, Display Colour, Different Ages, Colour Matching Functions

\section{Introduction}

In our daily life, almost all products are inseparable from the "colour", whether it is electronic products or clothing, books and other designs, which will bring different colour sense. With the development of modern colour science and technology, there are more and more ways and devices to reproduce colour, which will arouse different colour perceptions for the same observer under different light sources (named Illumination metamerism), or for different observers under the same light source (named observer metamerism) (ASTM,2012). In recent years, with the development of the devices with narrow band and wide colour gamut, it is obvious for observer metamerism, which is mainly caused by the differences of the spectral response of the observer, influences by age and viewing field.

In 2006, CIE(CIE,2006) proposed CIE2006 to calculate the colour matching functions (CMFs) of the observers aged from $20-80$ and the filed sizes between $1^{\circ} \sim 10^{\circ}$. Asano(Asano,2015), Ruili He (Ruili $\mathrm{H}, 2018$ ) have organized 58 observers aged from18-69 and 56 observers aged from 18-74 to evaluate the colour differences of metameric samples using the psychological method of paired-comparison, respectively. It was found that the young observer and the old observer had quite different results. As for the application of colour harmony for different aged observers, the results from Li-Chen Ou(L.C, Ou,2012) and Min Huang (Min. H,2017) had also revealed significant differences between young and old observers.

In order to investigate the colour difference discrimination of young and old observers, 5 colour centers (gray, red, yellow, green, blue) recommended by $\mathrm{CIE}(\mathrm{CIE}, 1978)$ were selected to present on the target display, 47 observers(30 young and 17 old observers) with normal colour vision were organized to carry out the colour matching experiments on the matched display. The results can be used to test the colour difference discrimination between young and old observers and the spectral data can be used to examine the colour matching functions for the observers with different age. 


\section{Experimental}

\subsection{Display Device}

In our experiment, two displays with different spectral primaries: APPLE IPAD2018 AIR2 (hereinafter referred to as IPAD) and QUATO-220ex (hereinafter referred to as QUATO) were selected and their correlated colour temperature as well as brightness were set as $5000 \mathrm{~K}$ and $120 \mathrm{~cd} / \mathrm{m}^{2}$ separately. The spectral power distributions of RGB three primaries and the white point were measured by Photo Research 655 spectroradiometer (hereinafter referred to as PR655) at a distance of $60 \mathrm{~cm}$, their normalized spectral power distributions are illustrated in Figure1. In the colour matching experiment, IPAD was used as the target device while QUATO display as the matching device.

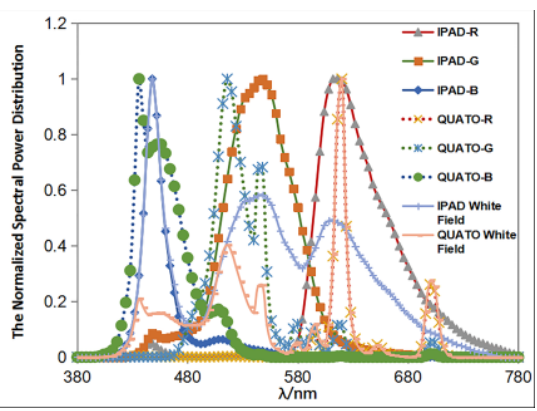

Figure 1 - The normalized spectral power distributions of red, green, blue and white points of two displays

\subsection{Colour Stimuli}

Five colour centers (gray, red, yellow, green, blue) recommended by CIE were chosen as the target colours(A. R. Robertson,1978), which were distributed uniformly in the CIELAB colour space and without high saturation. They were reproduced respectively on the center of IPAD and the spectral power distributions of each colour stimulus measured by PR655 were used to calculate their colourimetric values together with CIE1964 colour matching function. The obtained colourimetric values and the CIE recommended values are shown in Table 1.

Table 1 - CIE recommended values and experimental colourimetric values of five colours

\begin{tabular}{|c|c|c|c|c|c|c|c|c|c|c|c|}
\hline & \multicolumn{4}{|c|}{ CIE recommended values } & \multicolumn{4}{|c|}{ Experimentalcolourimetricvalues } & \multirow{2}{*}{} \\
\cline { 2 - 12 } & $L_{10}^{*}$ & $a_{10}^{*}$ & $b_{10}^{*}$ & $C_{10}^{*}$ & $h_{10, a b}^{*}$ & $L_{10}^{*}$ & $a_{10}^{*}$ & $b_{10}^{*}$ & $C_{10}^{*}$ & $h_{10, a b}^{*}$ & \\
\hline Gray & 62 & 0 & 0 & 0,0 & 0,0 & 63,0 & $-0,5$ & 0,6 & 0,8 & 127,0 & 1,3 \\
\hline Red & 44 & 37 & 23 & 43,6 & 31,9 & 45,9 & 37,7 & 23,3 & 44,3 & 31,7 & 2,0 \\
\hline Yellow & 87 & -7 & 47 & 47,5 & 98,4 & 88,6 & $-6,9$ & 46,3 & 46,8 & 98,4 & 1,8 \\
\hline Green & 56 & -32 & 0 & 32,0 & 180,0 & 57,0 & $-27,1$ & 0,7 & 27,1 & 178,5 & 5,0 \\
\hline Blue & 36 & 5 & -31 & 31,4 & 279,1 & 37,1 & 3,1 & $-31,1$ & 31,2 & 275,7 & 2,2 \\
\hline
\end{tabular}

\subsection{Observers}

47 observers with normal colour vision were organized to participate in the colour matching experiment, which including 30 young observers aged from 20 to 27 (14 male and 16 female) and 17 old observers aged from 61 to 74 ( 5 male and 12 female). The average age of young observers was 23 and all of them are college students majoring in printing engineering at Beijing Institute of Graphic Communication, with basic knowledge of colour science. The old observers were retired people and with an average age of 68 . 


\subsection{Colour Matching Experiment}

The colour matching experiment was carried out in a dark room. each display would be warmed up for 120 minutes before starting the real experiment.In case of the effectof the black screen on human colour perception during the experiment, a black mask $\left(\mathrm{Y}_{10}=4.72\right)$ with an open window (the size is $9 \mathrm{~cm} \times 9 \mathrm{~cm}$ ) was used to cover the entire screen and the colour stimulus was presented through the open window of the black mask. The experimental setup is shown in Figure2.

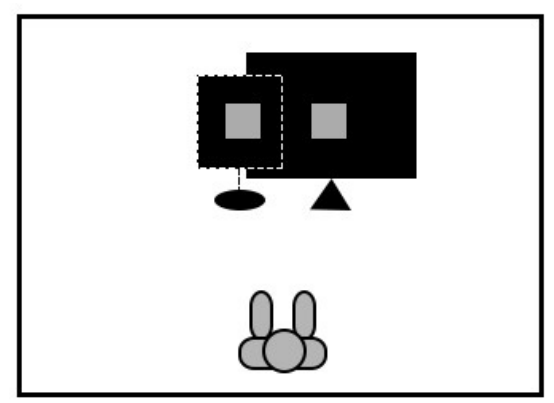

Figure 2 - Diagram of colour matching experiment

During the colour matching experiment, the observer was sitting in front of the display screen at a distance of about $60 \mathrm{~cm}$, with the eyes at the same horizontal line as thecolour block. For the colourstimulus displayed on the IPAD, the observer needs to adjust the RGB three channels on the QUATO display to match the colours presented on the center of IPAD. When each observer completes a matching of colour stimulus, the spectral power distributions of the target colour and the matched colour on two display devices were measured by PR655 timely. It took about 5 minutes for an observer to match a colourstimulus on the QUATO monitor. In order to avoid visual fatigue, observers need to complete a group ofcolour matching experiments at different time intervals.

Among the 30 young observers, 5 and 2 peoplehave made six repetitions and two repetitions respectively, while the other 23 observers performed a group of experiments once. Therefore, 57 groups of colour matching data were collected for young observers. For the 17 old observers, they repeated the experiments four times except one person made three repetitions and finally 67 groups of colour matching data were collected for old observers. In total, there are 620 colour matching data sets (124 groups x 5 colour centers) during the colour matching experiments.

\section{Analysis and Discussions}

\subsection{Observer Variability}

MCDM (the Mean colour Difference from the Mean) was used to study the observer variability for the matched colourdata, expressed as Eq. (1)(Oicherman.B,2010). Inter-observer variability can be defined as the deviation between thecolourimetric values matched by observers and the average matching results of all observers. Intra-observer variability refers to the deviation between the colourimetric values matched by an observer and the average results of all repeated matching colourimetric values of that observer.

$$
M C D M=\frac{\sum_{i=1, N}\left[\operatorname{CIELAB(}\left(C_{i}, C_{a v e}\right)\right]}{N}
$$

When calculating the inter-observer variability,

$N$ is the number of observers;

$C_{i}$ is the CIELAB colourimetric values matched by each young / old observer; 
$C_{\text {ave }}$ is the average CIELAB colourimetric values matched by all young / old observers.

When calculating the intra-observer variability,

$N$ is the number of repeated times;

$C_{i}$ is the CIELAB colourimetric values of each colour match for the observer;

$C_{a v e}$ is the average results of all repeated matching colourimetric values for the observer.

The smaller the MCDM value is, the smaller the deviation of the matching colour data is. It can be concluded that the accuracy and repeatability of young observers (1,91 and 3,56 respectively) are lower than those of older observers (3,56 and 5,43 respectively), which indicates that young observers performed more accurately than old observers in colour matching experiments between different displays.

\subsection{Colourimetric values}

The CIE L ${ }^{*}{ }_{10} \mathrm{a}^{*}{ }_{10} \mathrm{~b}^{*}{ }_{10}$ colourimetric values of the target colours and matching colours for young and old observers obtained from the colour matching experiments were plotted in the $\mathrm{a}^{*}{ }_{10} \mathrm{~b}^{*}{ }_{10}$ plane and $L^{*}{ }_{10} C^{*}{ }_{10}$ plane, as shown in Figure3(a) and Figure $3(b)$.

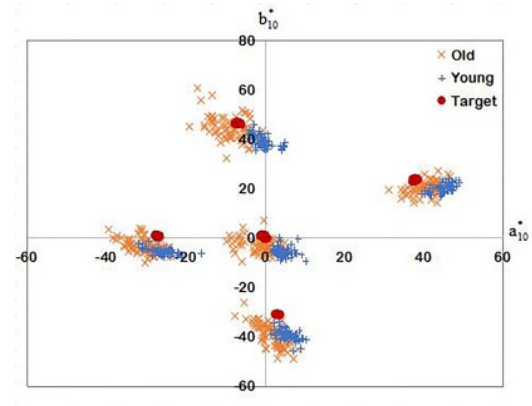

(a) $a^{*}{ }_{10} b^{*}{ }_{10}$ plane

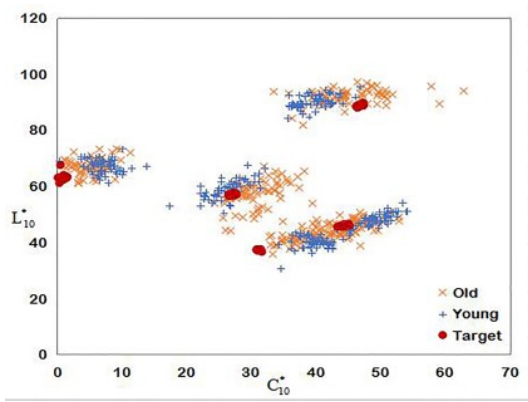

(b) $\mathrm{L}^{*}{ }_{10} \mathrm{C}^{*}{ }_{10}$ plane

Figure 3 - The distributions of targetand matched colours for young and old observers

In Figure3, the assembled red circles refer to the target colours displayed on the IPAD during the whole experiment, indicating that the stability of the reproduced target colour centers on IPAD is good. The yellow marks refer to the distributions of all colours on the QUATO display matched by old observers while the blue crosses refer to the colour distributions matched by young observers. Compared with the old observers' matching results, the distributions of matching colours for young observers are more concentrated, indicating that the young observers have smaller individual differences.

It can be seen from Figure3(a), the matching colours from young observers are shifted to the bottom-right of the target colour on the $a^{*}{ }_{10} b^{*}{ }_{10}$ plane while the colours matched by old observers are much closer to the target colours, even though the data distributed more dispersedly. In addition, as shown in Figure3(b), the colours matched by old observers presented larger variations in the direction of $\mathrm{C}^{*}{ }_{10}$ axis, which indicates that the old observers have a larger colour difference discrimination and a lower sensitivity in terms of the variations of chroma. Moreover, there is no significant difference in the direction of $L^{*} 10$ axis for young and old observers' matching colours.

Furthermore, a computational colour-matching simulation was performed to examine the differences from a group of observers with different age. The simulative workflow was the one proposed by Asano et al(Asano,2015), using as reference samples the 5 colours displayed on the IPAD viewed by CIE1964 observers and as matching colours those from the QUATO display. 108 CMFs were considered for simulations considering the average age of the young and old observers in our experiments, the average age of 23 and 68 from young and old 
observers were taken in CIE2006. Figure4 illustrates the distributions of matching colours on CIELAB plane calculated by CIE1964, 108 colour matching functions correspondingly.

As shown in Figure4, the black points are the colorimetric values calculated by CIE 1964 CMFs for the $5 \mathrm{CIE}$ recommended colour centers displayed on the IPAD. The red region is the colorimetric values calculated by the 108 colour matching functions mentioned before. The blue and green points represent the colours matched by the observers aged 23 and 68 calculated by CIE2006.

Comparing the Figure 3 and Figure4, it can be clearly seen that the simulated results from the colour matching experiments are consistent with those obtained from the experimental results.

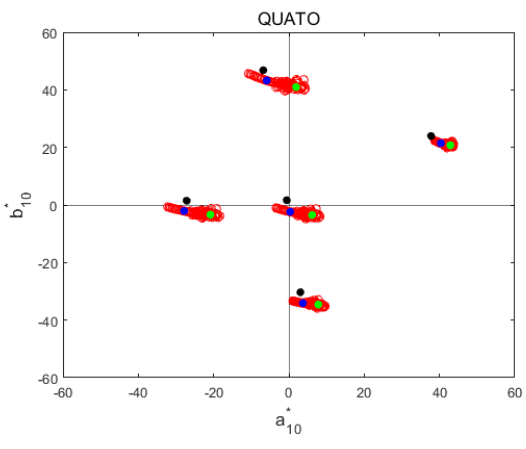

(a) $a^{*}{ }_{10} b^{*}{ }_{10}$ plane

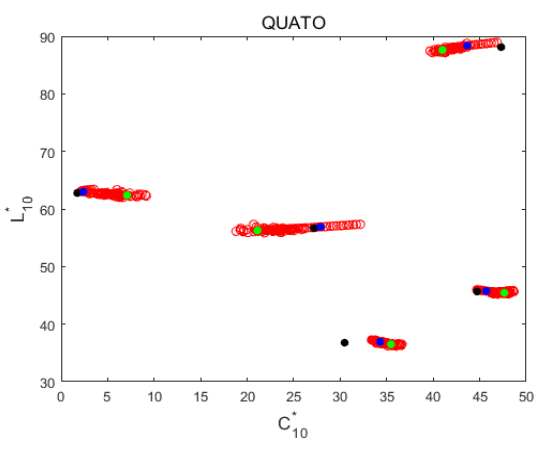

(b) $\mathrm{L}^{*}{ }_{10} \mathrm{C}^{*}{ }_{10}$ plane

Figure 4 - Scattering distributions of simulative calculation of different CMFs

All data of the old observers shift to the direction of $-a^{*}{ }_{10}$ axis, which may be caused by the different spectral response of the retinal cones from the old observers, which mainly occurred at the regions of macular.

\section{Conclusions}

In order to study the difference of colour difference discrimination between young and old observers, the colour matching experiment based on the 5 colour centers recommended by $\mathrm{CIE}$ was carried out using two display devices (IPAD and QUATO) with different primary colour spectral bandwidths. 30 young and 17 old observers with normal colour vision were organized to carry out the colour matching experiments with the method of binocular simultaneous. The spectral data of the target and the matched colours were collected by PR655 spectroradiometer, and CIE 1964 CMFs were also used together to calculate the CIELAB colourimetric values of the target colours on IPAD and matched colours on QUATO. The results indicate that distributions of colourimetric values from the observers with different ages are quite different, distributed diversely on $a^{*} 10 b^{*} 10$ plane and $L^{*} 10 C^{*} 10$ plane. Compared with young observers, old observers have greater individual differences in colour matching and the colour discrimination difference is more obvious. In both the colour matching experiment and the simulated computation, it was found that the colourimetric data matched by old observers tended to shift toward-a ${ }^{*} 10$ axis.

\section{References}

A. R. Robertson.1978. CIE Guidelines for Coordinated Research on Colour-Difference Evaluation. Colour Research and Application, 3(3): 149-151.

Asano Y. 2015. Individual colourimetric observers for personalized colour imaging. Rochester, Rochester Institute of Technology.

CIE 2006. CIE 170-1-2006. Fundamental Chromaticity Diagram with Physiological Axes - Part I.Vienna,Austria: CIE. 
E284 - 95a. 2012. Standard Terminology of Appearance. USA: ASTM.

Li-Chen Ou, et al.2012. Age Effects on Colour Emotion, Preference, andHarmony. Colour Research and Application, 37(2): 92-105.

Min H, et al. 2017. Colour Harmony in Two - Piece Garments. ColourResearch \& Application, 42(4): 498-511.

Oicherman B, et al. 2010. Effect of Observer Metamerism on Colour Matching of Display and Surface Colours. Colour Research \& Application,33(5): 346-359.

Ruili H, et al. 2018. Colour-Difference Discrimination Between Young and Old Observers. Laser \& Optoelectronics Progress, 55(3):033301.(in Chinese) 